\title{
OPERATOR ALGEBRAS AND DUAL SPACES
}

\section{R. CREIGHTON BUCK}

1. An isomorphism theorem. In this paper we discuss a particular method of introducing a convolution product into certain topological linear spaces so that they become algebras. The space in each case will be essentially the dual space of a linear space of continuous functions, and arises in the study of general group algebras and in the theory of distributions.

Let $G$ be a topological group, written additively but not necessarily abelian. Let $C^{*}(G)$ be the linear space of all complex-valued continuous functions on $G$. For $x \in G$ let $U_{x}$ be the left translation operator which sends a function $\phi$ into the function $U_{x} \phi=\phi_{x}$ whose value at $t$ is $\phi(x+t)$. Let $X$ be a subspace of $C^{*}(G)$, topologized by a locally convex topology $\tau$. Let $L(X)$ be the space of linear functionals on $X$ which are $\tau$ continuous on all $\tau$ bounded sets of $X$. Let $B(X)$ be the algebra of linear operators on $X$ which are $\tau$ bounded and $\tau$ continuous on the $\tau$ bounded sets of $X$. These contain the usual dual space $X^{\prime}$ and endomorphism algebra $E(X)$ and in certain important cases coincide with them, for example if $\tau$ is a norm topology. The operators $U_{x}$ form a group $G_{0}$ of operators on $C^{*}(G)$ anti-isomorphic to $G$. If $X$ is invariant under $G_{0}$ and $\tau$ suitable, we may regard $G_{0}$ as part of $B(X)$. In this case, let $B^{\circ}(X)$ be the set of all $T$ in $B(X)$ which commute with $G_{0} . B^{0}(X)$ is evidently a subalgebra of $B(X)$. Our first theorem shows that under certain conditions $L(X)$ and $B^{0}(X)$ are isomorphic as linear spaces, thus permitting us to introduce an algebraic structure into $L(X)$.

We first define two fundamental mappings between operators and functionals. For any operator $T$ in $B(X)$, let $F$ be a functional on $X$ defined by

$$
F(\phi)=T(\phi)(0) .
$$

For a functional $F$ in $L(X)$, let $T$ be an operator on $X$ defined by

$$
T(\phi)=\psi \text { where } \psi(x)=F\left(U_{x} \phi\right) .
$$

THEOREM 1. If the functional $F$ defined by (1) is in $L(X)$ for each $T$ in $B^{0}(X)$, and if $T$ defined by (2) is in $B(X)$ for each $F$ in $L(X)$, then (1) (or (2)) defines an isomorphism of $B^{\circ}(X)$ and $L(X)$ as linear spaces.

Presented to the Society, April 27, 1951; received by the editors April 3, 1951 and, in revised form, January 11, 1952. 
We need show only that (1) and (2) are inverse, and that $T$ defined in (2) lies in the subalgebra $B^{0}(X)$. For the latter, $T U_{y}(\phi)(x)$ $=F\left(U_{x} U_{y} \phi\right)=F\left(U_{y+x} \phi\right)=\psi(y+x)=U_{y}(\psi)(x)=U_{y} T(\phi)(x)$. Since this holds for all $x$ and $\phi, T U_{y}=U_{y} T$, and $T \in B^{0}(X)$. Given $T$ in $B^{0}(X)$, define $F$ by (1), and then $T^{\prime}$ by (2). Then, $T^{\prime}(\phi)(x)=F\left(U_{x} \phi\right)$ $=T\left(U_{x} \phi\right)(0)=U_{x} T(\phi)(0)=T(\phi)(x)$, and $T^{\prime}=T$. Thus, (1) is a oneto-one embedding of $B^{0}(X)$ in $L(X)$. Given $F$ in $L(X)$, define $T$ by (2), and $F^{\prime}$ by (1). For any $\phi, F^{\prime}(\phi)=T(\phi)(0)=F\left(U_{0} \phi\right)=F(\phi)$, and $F^{\prime}=F$.

Since $B^{0}(X)$ is also an algebra, the operation of operator multiplication induces a multiplication of functionals in the space $L(X)$. Given two functionals $F_{1}$ and $F_{2}$, let $T_{1}$ and $T_{2}$ be their images in $B^{0}(X)$. Then, $F_{1} * F_{2}$ is defined to be the functional corresponding to $T_{1} T_{2}$. Under *, $L(X)$ becomes an algebra isomorphic to $B^{0}(X)$. The identity operator $I$ corresponds to the functional $\delta$ defined by $\delta(\phi)$ $=I(\phi)(0)=\phi(0)$; this "dirac function" is then the unit for $L(X)$. The operation * can be given a more explicit description. For the value of $F$ at $\phi$, let us introduce the alternate notation $F(\phi(t) d t)$. Then, the function $\psi$ of (2) may be given by $\psi(x)=F(\phi(z+t) d t)$ and $\left(F_{1} * F_{2}\right)(\phi)$ is readily seen to be $F_{1}\left(F_{2}(\phi(x+t) d t) d x\right)$. The * product can thus be considered as a generalization of the familiar convolution of functions and of measures.

The remaining sections will deal with special choices of $X$ and $\tau$ under which the hypotheses of Theorem 1 may be established. Explicitly, it must be shown that $F$ in (1) is $\tau$ continuous on $\tau$ bounded sets, that $\psi$ in (2) lies in $X$, and that the mapping $\phi \rightarrow \psi$ is $\tau$ bounded and $\tau$ continuous on $\tau$ bounded sets. In addition, $X$ must be invariant under left translation, and the operators $U_{x}$ bounded and continuous.

2. Spaces of continuous functions. We distinguish certain special subspaces of $C^{*}(G)$. The first, $C^{f}(G)$, is the set of functions $\phi$ having the property that for each, there is an open set $V \subset G$ such that $\phi$ is bounded on $V$ and all of its translates. $C(G)$ is the set of all $\phi$ which are bounded on $G$, and $C_{u}(G)$ is the set of $\phi$ in $C(G)$ which are uniformly continuous on $G$. On each of these, several familiar topologies may be imposed. We shall use four, labeled as $\omega, \kappa, \beta$, and $\sigma$. For convenience, set $\|\phi\|_{S}=\sup _{x \in S}|\phi(x)|$ for $S \subset G$. The topology $\omega$ is that of pointwise convergence on $G$, and is the smallest (weakest) of the topologies. The largest is $\sigma$ which is that of uniform convergence on $G$. The former is applicable to $C^{*}$ and its subspaces, and the latter to $C . \sigma$ is a norm topology, defined by $\|\phi\|_{\sigma}$ and, under it, $C$ and $C_{u}$ are Banach spaces. When $G$ is locally compact, two additional topologies will be used. The $\kappa$ topology is the usual one of 
compact convergence, and is applicable to $C^{*}=C^{*}$, and more especially to $C$. It may be defined by the collection of pseudonorms $\|\phi\|_{K}$ for compact sets $K \subset G$. It is not complete. The $\beta$ topology, or "strict" topology, may be defined as follows. Let $g$ be any non-negative function in $C(G)$ which vanishes at infinity, and set $\|\phi\|_{0}=\|\phi g\|_{G} . \beta$ is the convex topology defined by this collection of pseudonorms. It is applicable to $C$ and, as the following lemma shows, it has some of the better features of both $\sigma$ and $\kappa$. It is similar to a topology introduced by Beurling [1].

LEMma. The $\beta$ topology on $C(G)$ has the following properties: (i) $\kappa \leqq \beta$ $\leqq \sigma$, (ii) $\beta$ and $\sigma$ have the same bounded sets, (iii) $\beta$ and $\kappa$ coincide on $\sigma$ bounded sets, (iv) $\beta$ is a sequentially complete topology.

Since $\|\phi\|_{0} \leqq\|\phi\|_{G}\left\|_{g}\right\|_{G,} \beta$ is smaller than $\sigma$. Given a compact set $K \subset G$, choose $g_{0}$ so that $g_{0}(x)=1$ on $K$. Then, $\|\phi\|_{K} \leqq\|\phi\|_{\sigma_{0}}$ and $\kappa \leqq \beta$. Any uniformly bounded set is strictly bounded. Conversely, if $S \subset C(G)$ is strictly bounded but were not uniformly bounded, $\phi_{n} \in S$ and $x_{n} \in G$ could be chosen with $\left|\phi_{n}\left(x_{n}\right)\right|=\lambda_{n}$ and $\lim \lambda_{n}=\infty$. The sequence $\left\{x_{n}\right\}$ is convergent in $G$ to infinity, and we may choose $g$ so that $g\left(x_{n}\right)=\lambda_{n}^{-1 / 2}$. Then, $\left\|\phi_{n}\right\|_{0} \geqq \lambda_{n}^{1 / 2}$, contradicting the strict boundedness of $S$. To prove (iii), let $S$ be a subset of $C(G)$ such that $\|\phi\|_{G} \leqq M$ for all $\phi \in S$, and let $\phi_{0}$ be in the $\kappa$ closure of $S$. Given $\epsilon>0$ and any $g$ choose $K$ so that $\|g\|_{G-K}<\epsilon$. Then,

$$
\begin{aligned}
\left\|\phi-\phi_{0}\right\|_{\sigma} & \leqq\left\|\phi-\phi_{0}\right\|_{K}\|g\|_{G}+\left\|\phi_{\phi}-\phi_{0}\right\|_{G}\|g\|_{G-K} \\
& \leqq\left\|\phi-\phi_{0}\right\|_{K}\|g\|_{G}+\epsilon\left\|\phi-\phi_{0}\right\|_{G} .
\end{aligned}
$$

If $\phi \in S$ and $\left\|\phi-\phi_{0}\right\|_{K}<\epsilon$, then

$$
\left\|\phi-\phi_{0}\right\|_{0} \leqq \epsilon\|g\|_{G}+\left(M+\left\|\phi_{0}\right\|_{G}\right) \epsilon
$$

and $\phi_{0}$ lies in the strict closure of $S$. Finally, a $\beta$ Cauchy sequence is $\beta$ bounded and hence $\sigma$ bounded, and therefore $\kappa$ and hence $\beta$ convergent to a member of $C(G)$.

It is evident from the above that a sequence $\left\{\phi_{n}\right\}$ is $\beta$ convergent if and only if $\kappa$ convergent and uniformly bounded. Briefly, $\beta$ may be described as the convex topology on $C(G)$ obtained from the pseudotopology of $\kappa$ convergent uniformly bounded sets.

We shall set $\|F\|=\sup |F(\phi)|$, taken over all $\phi$ in $X$ with $\|\phi\|_{G} \leqq 1$. Since a uniformly bounded set is bounded in each of the topologies, $\|F\|$ is finite for every $F$ in $L(X)$. Similarly, if $T \in B(X)$, we set $\|T\|$ =sup $\|T(\phi)\|_{G}$, taken as above. We cannot immediately conclude that $\|T\|<\infty$, although this follows for $T \in B^{\circ}(X)$ from the next theorem. 
ThEOREм 2. Under the convolution product * and the norm $\|$, the spaces $L(X)$ become topological algebras in the following cases: (i) $X$ $=C^{\ddagger}(G), \tau=\omega$, (ii) $X=C(G), \tau=\kappa$ or $\beta$, (iii) $X=C_{u}(G), \tau=\sigma$.

We shall use Theorem 1 to establish a norm preserving isomorphism between $B^{0}(X)$ and $L(X)$ in each case. We first observe that each $X$ is invariant under $U_{x}$. Moreover, $\left\|U_{x} \phi\right\|_{S}=\|\phi\|_{x+S}$ and $\left\|U_{x} \phi\right\|_{0}$ $=\|\phi\|_{h}$ where $h=U_{-x} g$. Thus, in each case, the operators $U_{x}$ lie in $B(X)$. Assume now that $T \in B^{0}(X)$ and define $F$ by $(1) ; F(\phi)$ $=T(\phi)(0)$. Since $T$ is $\tau$ continuous on $\tau$ bounded sets and since $\omega \leqq \tau, F$ is in $L(X)$. Consider the mapping $x \rightarrow U_{x} \phi$ of $G$ into $X$. If $\phi$ is unbounded, but lies in $C^{f}$, there is a neighborhood $V$ of 0 in $G$ such that the set of all $U_{x} \phi$ for $x \in V$ is an $\omega$ bounded subset. Since $\phi$ itself is continuous, the mapping $x \rightarrow U_{x} \phi$ is continuous at 0 . If $F \in L(X)$ in case (i), $F$ is continuous on $\omega$ bounded sets, and $\psi(x)$ $=F\left(U_{x} \phi\right)$ is continuous for $x \in V$. Moreover, $F$ is bounded on $\omega$ bounded sets so that $\psi(x)$ is bounded on $V$. Similarly, $\psi$ is bounded and continuous on each translate of $V$, and thus lies in $C^{\star}$. We turn next to case (ii). Since $\phi \in X$ is now bounded, the set of all $U_{x} \phi$ for $x \in G$ is a $\sigma$ bounded set, and thus $\kappa$ and $\beta$ bounded. Since $\kappa$ and $\beta$ coincide on $\sigma$ bounded sets, we can discuss these together. If $K \subset G$ is compact, then $\phi$ is uniformly continuous on $K$ and the mapping $x \rightarrow U_{x} \phi$ is continuous; as before, for any $F$ in $L(C), \psi(x)$ is continuous, and since $|\psi(x)| \leqq\|F\|\|\phi\|_{G}, \psi$ is bounded and therefore lies in $C(G)=X$. Finally, in case (iii) $\phi$ is uniformly continuous on all of $G$ and $x \rightarrow U_{x} \phi$ is uniformly $\sigma$ continuous so that $\psi(x)$ is uniformly continuous and therefore in $C_{u}$. In each case, we have verified that (2) defines a linear transformation $T$ of $X$ into itself. It remains to show that $T$ is appropriately continuous and bounded. Since $\|T(\phi)\|_{G}=\|\psi\|_{G} \leqq\|F\|\|\phi\|_{G}$ we see that, in all cases, $\|T\| \leqq\|F\|<\infty$. Since $T$ is then $\sigma$ continuous on $\sigma$ bounded sets, the proof of case (iii) is complete. Case (i) is also easily disposed of. For fixed $x, F U_{x}$ is continuous on $\omega$ bounded sets, and it then follows at once that $T$ is $\omega$ continuous on $\omega$ bounded sets. Similarly, since $F$ must be bounded on $\omega$ bounded sets, $T$ carries $\omega$ bounded sets into $\omega$ bounded sets. Case (ii) requires slightly more argument. Here $X=C(G)$ and $\tau=\kappa$ or $\beta$. We discuss $\kappa$ first. Let $S$ be a $\kappa$ bounded set in $X$ containing 0 , and let a compact set $K \subset G$ and $\epsilon>0$ be given. Let $S^{*}=\left\{\right.$ all $U_{x} \phi \mid x \in K$, $\phi \in S\} . S^{*}$ is also $\kappa$ bounded. Since $F \in L(X)$ is $\kappa$ continuous on $S^{*}$, choose $K_{1}$ and $\delta$ so that $\phi \in S^{*}$ and $\|\phi\|_{K_{1}}<\delta$ imply $|F(\phi)|<\epsilon$. Set $K_{2}=K+K_{1}$. Then, if $x \in K,\left\|U_{x} \phi\right\|_{K_{1}} \leqq\|\phi\|_{K_{2}}$ so that $\phi \in S$ and $\|\phi\|_{K_{2}}<\delta$ imply that $\left|F\left(U_{x} \phi\right)\right|<\epsilon$ for all $x \in K$. Rephrased, this states that if $\phi \in S$ and $\|\phi\|_{K_{2}}<\delta$, then $\|\psi\|_{K}<\epsilon$, proving that $T$ is 
$\kappa$ continuous on $\kappa$ bounded sets. We have also proved that the image of $S$ is bounded, since $|F(\phi)| \leqq A$ holds for all $\phi \in S^{*}$, so that if $\phi \in S,\|\psi\|_{K} \leqq A$. The argument for $\tau=\beta$ is implicit in the discussion above, since the set $S$ being $\beta$ bounded is $\sigma$ bounded, and so is $S^{*}$, and $\beta$ and $\kappa$ agree on such sets. Finally, to show that the correspondence established in (1) and (2) is norm preserving, we note that we have shown above that $\|T\| \leqq\|F\|$. Since $|F(\phi)|=|T(\phi)(0)|$ $\leqq\|T(\phi)\|_{G}$, we also have $\|F\| \leqq\|T\|$.

It perhaps should be be pointed out that the space $L(X)$ in case (ii) contains an image of the usual $L^{1}$ algebra of $G$. If $f \in L^{1}(G)$, then the functional $F$ defined by $F(\phi)=\int_{G} f \phi$ is in $L(C(G))$ if $\tau$ is chosen as $\beta$, although not if $\tau=\kappa$. This approach, using case (iii), has been discussed elsewhere [2].

3. The space of distributions. Let $G$ be the additive group of reals, with the usual locally compact topology. Let $\varepsilon$ be the subspace of $C^{*}(G)$ composed of the infinitely differentiable functions, and let $\mathscr{D}$ be the subspace of those $\phi$ which have compact support, that is, vanish off a compact set. On $\varepsilon$ we impose the topology $\tau$ generated by the pseudonorms $\|\phi\|_{K, p}=\left\|D^{p} \phi\right\|_{K}$ where $D$ is the differentiation operator, and $K$ is a compact set. Clearly $\tau$-lim $\phi_{\alpha}=0$ means merely that $x-\lim D^{p} \phi_{\alpha}=0$ for $p=0,1, \ldots$. Let $\varepsilon^{\prime}$ and $E(\varepsilon)$ be the usual dual space and endomorphism algebra of $\varepsilon ; E^{0}(\varepsilon)$ is again the subalgebra of those $T$ in $E(\varepsilon)$ which commute with the translation operators $U_{x}$.

TheOREM 3. $\mathcal{E}^{\prime}$ and $E^{0}(\mathcal{E})$ are isomorphic under the mappings (1) and (2).

We first observe that if $T$ is in $E^{0}\left(C^{*}\right)$ where $C^{*}$ has the $\kappa$ topology, and if $T$ leaves $\varepsilon$ invariant and commutes with $D, T$ is in $E^{0}(\varepsilon)$. As before, since $\omega \leqq \tau$, the functional $F$ defined by (1) is continuous and hence in $\mathcal{E}^{\prime}$. Given $F$ in $\varepsilon^{\prime}$ and defining $T$ by (2), $T$ is easily seen to belong to $E^{0}\left(C^{*}\right)$. With $\psi(x)=F\left(U_{x} \phi\right)$, we have $D \psi(x)$ $=\lim _{h \rightarrow 0} F\left(h^{-1}\left(U_{x+h}-U_{x}\right) \phi\right)$. For each $p, D^{p} \phi$ is continuous, and hence uniformly continuous on compact sets, and thus $\tau$ $\lim _{h \rightarrow 0} h^{-1}\left(U_{x+h}-U_{x}\right) \phi=U_{x} D \phi$. Since $F$ is $\tau$ continuous, $D \psi(x)$ $=F\left(U_{x} D \phi\right)$, and by induction, $D^{p} \psi(x)=F\left(U_{x} D^{p} \phi\right)$. This proves both that $\psi$ lies in $\varepsilon$ so that $T$ leaves $\varepsilon$ invariant, and that $T$ commutes with $D$. By the remark above, $T$ is in $E^{0}(\varepsilon)$.

The algebra $E^{0}(\mathcal{E})$ may be considered as the set of all $\kappa$ continuous operators on $C^{*}$ which carry $\mathcal{E}$ into itself and commute with $D$ and with translations. It contains the subalgebra of all differential operators with constant coefficients and of finite order, but does not 
contain such operators as $x^{2} D$. It also contains such integral transforms as $T(\phi)=\psi$ where

$$
\psi(x)=\int_{-\infty}^{\infty} H(x-t) \phi(t) d t
$$

where $H \in \mathcal{D}$. More generally, the theorem asserts that the general operator $T$ has the representation $T(\phi)=\psi$ with $\psi(x)=F\left(U_{x} \phi\right)$ for a unique $F$ in $\varepsilon^{\prime}$. This latter space is exactly the space of distributions having compact support (see [3, chap. 3]). As before, the multiplication of operators in $E^{0}(\varepsilon)$ induces a convolution product into $\varepsilon^{\prime}$ so that it too becomes an algebra. It is readily seen that this is the same product as that introduced in a different way by Schwartz. That portion of the theory of distributions relating to $\varepsilon^{\prime}$ can be transferred with some advantage to the operator algebra $E^{0}(\varepsilon)$. In place of the "derivative" of a distribution, the operator $D$ itself is present; if $F$ is the distribution corresponding to the operator $T$, the derivative $F^{\prime}$ corresponds to the operator $-D T$.

Turning now to the space $\mathcal{D}$, the importance of this subspace of $\mathcal{E}$ lies partly in the fact that, on it, $D$ is a one-to-one operator, whose range is the set of all $\phi$ with $\int_{-\infty}^{\infty} \phi=0$. We can consider $\mathcal{D}$ under the relative $\tau$ topology or under the Schwartz topology $\tau^{\prime}$ in which a convergent sequence must have a common supporting set. The dual space $\mathscr{D}^{\prime}$ is the space of all distributions; let $E(D)$ be the corresponding endomorphism algebra. The natural mappings (1) and (2) do not set up an isomorphism of $\mathscr{D}^{\prime}$ and $E^{0}(\mathscr{D})$; instead, $E^{0}(\mathscr{D})$ is embedded isomorphically in a proper part of $\mathscr{D}^{\prime}$. For example, the distribution 1 corresponds to the functional $F$ where $F(\phi)=\int_{-\infty}^{\infty} \phi$. The corresponding operator defined by (2) sends $\phi$ into $\psi$, with $\psi(x)=F(\phi)$ for all $x$; $\psi$ is a constant function, and lies in $\varepsilon$ but not $\mathcal{D}$. It is possible to obtain a space of operators which is isomorphic to the whole space $\mathscr{D}^{\prime}$. Consider $E^{0}(\mathcal{D} ; \varepsilon)$, the set of all continuous linear transformations of $\mathscr{D}$ into $\mathcal{E}$ which commute with $U_{x}$ for $x \in G$. The previous type of argument shows that (1) and (2) set up an isomorphism between $\mathscr{D}^{\prime}$ and $E^{0}(\mathscr{D} ; \varepsilon)$. The convolution of distributions can now be discussed in terms of the ordinary product of operators. Given $T_{1}$ and $T_{2}$ in $E^{0}(\Phi ; \varepsilon)$, the product $T_{1} T_{2}$ is an operator whose domain is $\mathscr{D} \cap T_{2}^{-1}(\mathscr{D})$. It corresponds to a distribution only if this domain is $\Phi$ (and thus if $T_{2}(D) \subset \mathcal{D}$ ) or, more generally, if the domain of $T_{1}$ can be expanded to include the range of $T_{2}$. For example, we recall that a distribution with compact support corresponds to an operator $T$ mapping $\mathcal{E}$ into $\mathcal{E}$, and $\mathcal{D}$ into $\mathscr{D}$. It is therefore possible to form the convolution of an arbitrary distribution and one with compact sup- 
port, in either order. (See [3, chap. 6].) In going from an operator to the corresponding distribution, only the action of the operator on $\mathscr{D}$ is considered; in this contraction, some properties of the operator algebra are lost, in particular convolution of distributions fails to be associative, although the product of operators of course is $[3$, chap. 6 , $\S 5]$. This suggests that it would perhaps be fruitful to study the general algebra of operators $T$ whose domains are subspaces of $\varepsilon$ containing $\mathcal{D}$, and whose ranges lie in $\mathcal{E}$.

\section{REFERENCES}

1. A. Beurling, Un theoreme sur les fonctions bornees, Acta Math. vol. 77 (1945) pp. $127-136$.

2. R. C. Buck, Generalized group algebras, Proc. Nat. Acad. Sci. U.S.A. vol. 36 (1950) pp. 747-749.

3. L. Schwartz, Theorie des distributions, Actualites Scientifiques et Industrielles, Paris, vol. 1, 1950, vol. 2, 1951.

UNIVERSITY OF WISCONSIN 